\title{
Assessment of Reversibility in Pulmonary Hypertension Related to Congenital Heart Disease by Using Biomarkers and Clinical Features
}

\author{
Liping Wang, Mingjie Zhang, Xi Chen, Yachang Pang, Jiaqi Liu and Zhuoming Xu* \\ Department of Cardiothoracic Surgery, Shanghai Children's Medical Center, School of Medicine, Shanghai Jiao Tong University, \\ Shanghai, 200127, China \\ *Corresponding Author: Zhuoming Xu. Email: zmxcicu@163.com
}

Received: 26 July 2021 Accepted: 24 September 2021

\begin{abstract}
Background: Reversibility of pulmonary hypertension (PH) is closely related to the treatment options for and prognosis of children with congenital heart disease. Objective: We combined patient-specific clinical features including diagnosis, age and echocardiographic results, and biomarkers of pulmonary vascular dysfunction to explore the noninvasive methods that can be used to accurately evaluate the reversibility of pulmonary hypertension in congenital heart disease (PH-CHD). Methods: Based on the preoperative systolic pulmonary arterial pressure (sPAP), 70 CHD patients were divided into normal, $\mathrm{PH}-\mathrm{CHD}$ suspected, and confirmed groups. Additionally, biomarkers of circulating endothelial cells (CECs), endothelin-1 (ET-1), and endothelial nitric oxide synthase (eNOS) were detected. Patients were categorized into reversible (RPH) and irreversible (IRPH) groups according to the sPAP 6 months after surgery. Risk stratification was performed according to the clinical features and biomarkers. Results: CECs and ET-1 levels in the confirmed group were significantly higher. eNOS was higher in the confirmed and suspected groups than that in the normal group. CECs in the IRPH group were significantly higher compared to the RPH group. No such intergroup differences were observed with respect to ET-1 and eNOS levels. The ROC curve showed that the risk stratification was of high diagnostic value to evaluate reversibility. Conclusion: The CECs, eNOS, and ET-1 were closely related with PH-CHD. CECs and risk stratification have high practical value in assessing the reversibility of $\mathrm{PH}-\mathrm{CHD}$.
\end{abstract}

\section{KEYWORDS}

Pulmonary hypertension in congenital heart disease; risk stratification; circulating endothelial cells; endothelin-1; endothelial nitric oxide synthase

\author{
Nomenclature \\ PH: $\quad$ Pulmonary hypertension \\ PH-CHD: Pulmonary hypertension in congenital heart disease \\ sPAP: $\quad$ Systolic pulmonary arterial pressure \\ CECs: $\quad$ Circulating endothelial cells \\ ET-1: $\quad$ Endothelin-1 \\ eNOS: $\quad$ Endothelial nitric oxide synthase \\ RPH: $\quad$ Reversible pulmonary hypertension \\ IRPH: Irreversible pulmonary hypertension
}




$\begin{array}{ll}\text { PVR: } & \text { Pulmonary vascular resistance } \\ \text { PAP: } & \text { Pulmonary artery pressure } \\ \text { NO: } & \text { Nitric oxide } \\ \text { TR: } & \text { Tricuspid regurgitation } \\ \text { ECHO: } & \text { Echocardiography } \\ \text { CHD: } & \text { Congenital heart disease } \\ \text { VSD: } & \text { Ventricle septal defect } \\ \text { PDA: } & \text { Patent ductus arteriosus } \\ \text { ASD: } & \text { Atrial septal defect } \\ \text { IAA: } & \text { Interrupted aortic arch } \\ \text { CoA: } & \text { Aortic coarctation } \\ \text { LVEF: } & \text { Left ventricular ejection fraction } \\ \text { ELISA: } & \text { Enzyme-linked immunosorbent assay } \\ \text { FSV: } & \text { Functional single ventricle } \\ \text { DORV: } & \text { Double outlet right ventricle } \\ \text { CAVSD: } & \text { Atrioventricular septal defect } \\ \text { TAPVC: } & \text { Complete anomalous pulmonary vein connection }\end{array}$

\section{Introduction}

Pulmonary hypertension in congenital heart disease (PH-CHD) is a severe condition threatening children's physical and mental health. With the development of medical technology, the diagnosis and treatment of PH-CHD have greatly improved in recent years. However, some patients, especially those with irreversible pulmonary hypertension (IRPH), still die of PH-CHD [1,2]. One of the main reasons is the lack of effective noninvasive methods to identify the reversibility of PH-CHD.

The typical pathophysiological changes of PH-CHD are divided into three stages [3]. In the early stage, hyperkinetic $\mathrm{PH}$ is characterized by increased pulmonary blood flow and normal pulmonary vascular resistance (PVR). If timely surgical repair is not performed, PH-CHD will progress to the second stage with increased pulmonary blood flow, pulmonary artery pressure (PAP), and PVR. In the late stage, irreversible pulmonary vascular remodeling occurs, with significantly increased PAP and PVR and decreased pulmonary blood flow, referred to as Eisenmenger syndrome. Throughout the process, the structure and function of the pulmonary vascular endothelium are closely related to the development of PH-CHD [4,5]. The vascular endothelium can directly sense changes in the hemodynamic and biochemical environment and release vasoactive substances to regulate vasomotion. Additionally, the process of irreversibility is silent. Therefore, structural and functional assessments of the pulmonary vascular endothelium have become important noninvasive methods of evaluating the reversibility of PH-CHD.

Abnormal pulmonary blood flow in PH-CHD patients impairs pulmonary vascular endothelial cells, which leads to an increase in circulating endothelial cells (CECs). Additionally, abnormal pulmonary blood flow stimulates vessels to release a variety of vasoactive substances, among which, endothelin 1 (ET-1) and endothelial nitric oxide synthase (eNOS) are closely related to the progression of PH-CHD [6-14]. The CEC count is relatively stable in healthy people but increases in subjects who undergo continued vascular endothelial damage [15]. When the vascular endothelium undergoes sustained damage, endothelial cells will shed into the bloodstream. Thus, CECs have become a reliable biomarker that can provide clues for the assessment of vessel damage. ET-1 is a potent endogenous vasoconstrictor peptide synthesized and released by endothelial cells. The concentration of ET-1 reflects the functional status of vascular endothelial cells and plays a pivotal role in the remodeling of the pulmonary vascular endothelium. eNOS is an important enzyme that catalyzes the generation of nitric oxide (NO). Its 
expression level directly determines the secretion of $\mathrm{NO}$ and plays an important role in maintaining function of the pulmonary vascular endothelium.

In addition to these biomarkers of endothelial injury, clinical studies have shown that the severity and reversibility of PH-CHD are also related to age, diagnosis, pulmonary hemodynamics, and clinical signs and symptoms [16-18]. The older the child is, the more complex the disease; the higher the PAP and PVR are, the greater the possibility of IRPH. For a more accurate assessment of disease status and to guide clinical diagnosis and treatment, a comprehensive evaluation should be carried out based on all indicators. In this study, we aimed to detect the concentrations of CECs, ET-1, and eNOS in CHD patients with different pathophysiologies and severities and combined them with various clinical features for risk stratification to assess the reversibility of $\mathrm{PH}-\mathrm{CHD}$ and to provide a noninvasive and accurate method to evaluate the reversibility of PH-CHD.

\section{Methods}

\subsection{Patient Selection}

This study was approved by the Institutional Health Research Ethics Board of the Shanghai Children's Medical Center, Shanghai Jiao Tong University School of Medicine (Approval No. SCMCIRB-Y2019044/ 2019) and written informed consent was obtained from the parent/legal guardian of participants. Seventy consecutive CHD patients who visited and underwent echocardiography (ECHO) at our hospital from September 2018 to July 2019 were enrolled in the present study. We excluded patients with lung disease and other diseases that could be an underlying cause of PH. To control the intergroup variation for better single-factor analysis, the echocardiography of all cases was performed by an attending and checked by a chief doctor who worked in the Department of Pediatric Cardiology. The diagnostic classification of patients is shown in Table 1 [19]. According to the 2015 ESC/ERS guidelines of PH, the patients were divided into three groups based on preoperative tricuspid regurgitation (TR) measured by ECHO [20]. Patients with TR $\leq 2.9 \mathrm{~m} / \mathrm{s}$ were classified as the normal group, $2.9 \mathrm{~m} / \mathrm{s}<\mathrm{TR} \leq 3.4 \mathrm{~m} / \mathrm{s}$ as the PH-CHD suspected group, and TR $>3.4 \mathrm{~m} / \mathrm{s}$ as the $\mathrm{PH}-\mathrm{CHD}$ confirmed group. Patients in the suspected and confirmed groups were followed-up for 6 months after the surgery. Those patients with TR $>3.4 \mathrm{~m} / \mathrm{s}$ and continuous deterioration 6 months after surgery or who died of postoperative $\mathrm{PH}$ were classified as the IRPH group, and the remaining patients formed the reversible pulmonary hypertension (RPH) group.

Table 1: Diagnostic classification in patients with CHD

\begin{tabular}{lll}
\hline Diagnosis & Cases & Classification [19] \\
\hline Simple CHD (VSD/PDA/ASD) & 21 & 1.4 .4 \\
Total anomalous pulmonary venous connection (TAPVC) & 10 & 2.4 \\
IAA/CoA + Simple CHD & 7 & 2.4 \\
Mitral valve malfunction & 3 & 2.4 \\
Cor triatriatum & 1 & 2.4 \\
Complete atrioventricular septal defect (CAVSD) & 8 & 5.4 \\
Double outlet of right ventricular (DORV) & 7 & 5.4 \\
Truncus arteriosus & 5 & 5.4 \\
Functional single ventricle (FSV) & 5 & 5.4 \\
Transposition of the great arteries (TGA) & 3 & 5.4 \\
\hline
\end{tabular}

Note: CHD, congenital heart disease; VSD, ventricle septal defect; PDA, patent ductus arteriosus; ASD, atrial septal defect; IAA, interrupted aortic arch; CoA, aortic coarctation. 
The left ventricular ejection fraction (LVEF) of CHD patients will increase because these patients experience pressure overload and cardiac hyperfunction. The LVEF of normal children is approximately $56 \%-78 \%$ under quiet conditions. In this study, the median LVEF was $69.9 \%(57.1 \%-84.5 \%)$, which was higher than that of the healthy population. For correct grouping, we used LVEF $=65 \%$ to normalize the velocity of TR.

\subsection{Biomarker Detection}

Blood samples of all patients were collected for biomarker detection before surgery. The CECs were quantified using flow cytometry. Briefly, $2 \mathrm{~mL}$ venous blood was collected in EDTA-coated tubes. Red blood cells were lysed using ACK Lysing Buffer (GibcoTM, NY, USA) per the manufacturer's instructions. Conjugated antibodies against CD45-per CP (BD Biosciences, NJ, USA), CD31-FITC (BD Biosciences, NJ, USA), and CD146-PE (BD Biosciences, NJ, USA) were used to identify CECs as previously described [21-23]. After a 40-min light-shielded incubation in a refrigerator at $4^{\circ} \mathrm{C}, \mathrm{CEC}$ counting was performed on a Fluorescence Activated Cell Separation (FACS) Canto-II flow cytometer (BD Biosciences, NJ, USA). The number of CECs was expressed as the ratio of CECs to mononuclear cells. Fig. 1 shows diagrams of CEC sorting by flow cytometry.
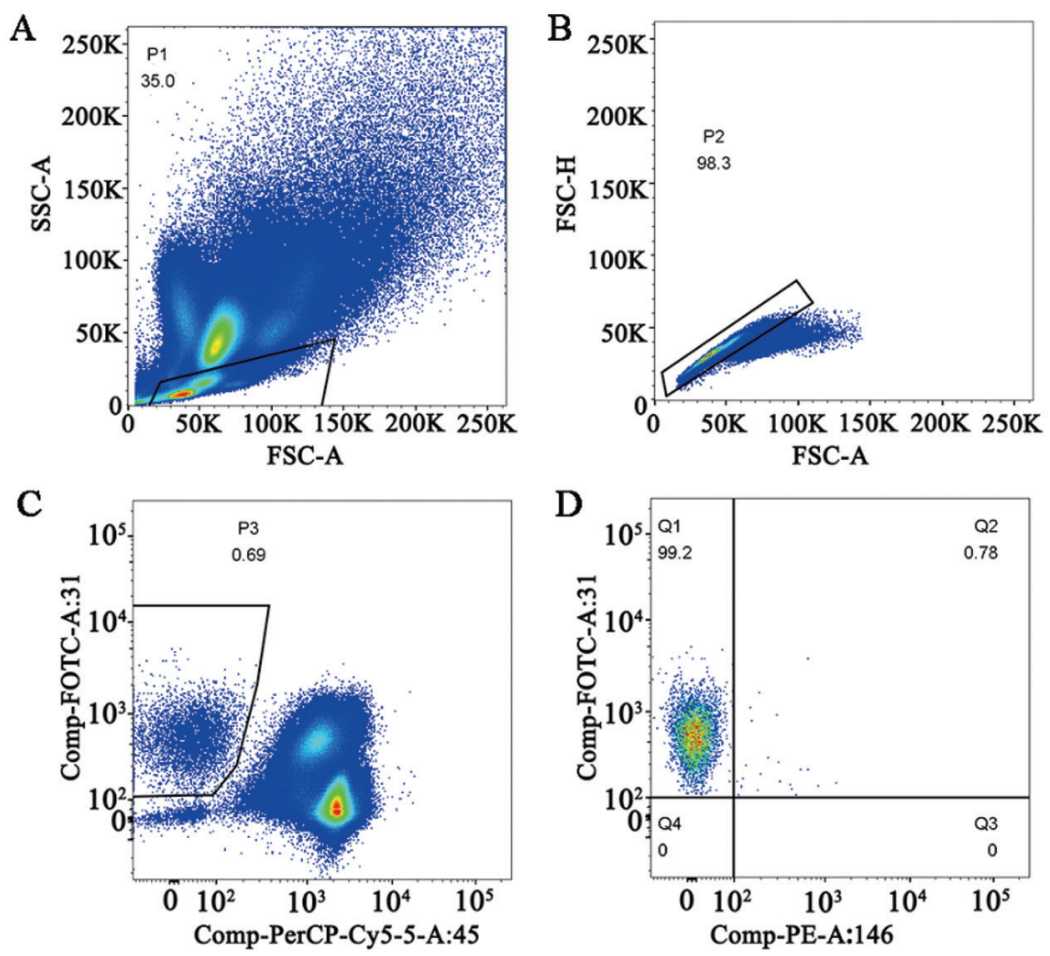

Figure 1: Diagrams of CECs sorting. A mononuclear cell screening (P1). B describes the gating on mononuclear without adhesion cells (P2). C selected CD31 + and CD45-cells (P3). D CD31 + and CD146 + co-expression cells, namely CECs (Q2)

Enzyme-linked immunosorbent assay (ELISA) was used to measure the concentration of ET-1 and eNOS (kits from Mlbio, Shanghai, China). Per the manufacturer's instructions, $50 \mu \mathrm{L}$ of each serum sample was used per well. After sequential antibody labeling, color reaction, and incubation, the optical density was measured to calculate the concentration of ET-1 and eNOS. 


\subsection{Risk Stratification}

Patient clinical features, including diagnosis, age, and ECHO evaluation, were collected and combined with the results of biomarkers for risk stratification. The risk score was decided based on relevant literature and represents the severity of PH-CHD $[3,16]$. Table 2 presents the scoring method of risk stratification.

Table 2: Risk stratification index and score in CHD patients

\begin{tabular}{llllllll}
\hline \multirow{2}{*}{ Score } & Diagnosis & \multicolumn{3}{c}{ Age } & ECHO & Biomarkers \\
\cline { 3 - 6 } & & ASD & $\begin{array}{l}\text { VSD/ } \\
\text { PDA }\end{array}$ & Complex CHD & FSV & \\
\hline 1 & ASD & $\leq 120 \mathrm{~m}$ & $\leq 12 \mathrm{~m}$ & $\leq 6 \mathrm{~m}$ & $\leq 3 \mathrm{~m}$ & $\begin{array}{l}\text { Confirmed } \\
\text { group }\end{array}$ & $\leq$ Cut-off value \\
2 & VSD/PDA & $>120 \mathrm{~m}$ & $12 \sim 24 \mathrm{~m}$ & $6 \sim 12 \mathrm{~m}$ & $3 \sim 6 \mathrm{~m}$ & & $>$ Cut-off value \\
3 & Complex CHD & & $24 \sim 60 \mathrm{~m}$ & $12 \sim 24 \mathrm{~m}$ & $6 \sim 12 \mathrm{~m}$ & \\
4 & FSV & $>60 \mathrm{~m}$ & $>24 \mathrm{~m}$ & $>12 \mathrm{~m}$ & \\
\hline
\end{tabular}

Note: ASD, atrial septal defect; VSD, ventricle septal defect; PDA, patent ductus arteriosus; CHD, congenital heart disease; FSV, functional single ventricle; ECHO, echocardiography.

\subsection{Statistical Analysis}

SPSS 23.0 (IBM, Armonk, NY, USA) was used for the statistical analysis. For non-normally distributed data, the dispersion degree was expressed using medians with interquartile ranges. The Mann-Whitney U test was used all intergroup comparisons. The Kruskal-Wallis $H$ test was used to compare the difference among multiple groups, and chi-square test was used for comparison of sex. $P<0.05$ was considered to indicate statistical significance.

\section{Results}

\subsection{Demographic Data}

Of the 70 cases, eight had no PH, 12 were included in the suspected group and 50 were included in the confirmed group. Four of the 62 children with suspected or confirmed PH died during hospitalization, and the remaining children were followed-up until 6 months after surgery. According to the follow-up results, 45 children were assigned to the RPH group and 14 to the IRPH group. Of the four patients who died, one died of postoperative pulmonary hypertension crisis; this patient was classified as having IRPH. In the IRPH group, four patients were diagnosed with functional single ventricle (FSV), three with ventricular septal defect (VSD), two with double outlet right ventricle (DORV), two with truncus arteriosus, two with atrioventricular septal defect (CAVSD), and one with complete anomalous pulmonary vein connection (TAPVC). Table 3 shows the demographic data of patients in different groups.

\subsection{Analysis of Biomarkers}

Statistical analysis showed that the levels of CECs and ET-1 were significantly higher in the confirmed group than in the normal and suspected groups (Table 4, Figs. 2A, 2B). eNOS levels in the confirmed and suspected groups were significantly higher than those in the normal group, and there was no significant difference between the confirmed and suspected groups (Table 4, Fig. 2C). Compared with the RPH group, the IRPH group showed a significant increase in CECs. However, there was no significant difference between the two groups with respect to ET-1 and eNOS levels (Table 4). The ROC curve showed that CECs were sensitive in diagnosing the reversibility of PH-CHD, but the specificity was relatively low (Fig. 3). 
Table 3: Patient demographics in different groups

\begin{tabular}{|c|c|c|c|c|c|c|c|}
\hline & \multicolumn{3}{|c|}{ Diagnosis } & \multirow[t]{2}{*}{$P$} & \multicolumn{2}{|c|}{ Reversibility } & \multirow[t]{2}{*}{$P$} \\
\hline & Group $1(n=8)$ & Group $2(n=12)$ & Group $3(n=50)$ & & $\mathrm{RPH}(\mathrm{n}=45)$ & IRPH $(n=14)$ & \\
\hline $\operatorname{Sex}(M / F)$ & $4 / 4$ & $7 / 5$ & $27 / 23$ & 0.964 & $26 / 19$ & $7 / 7$ & 0.609 \\
\hline Age (m) & $24(2 \sim 34)$ & $6(3 \sim 7)$ & $7(2 \sim 38)$ & 0.289 & $5(2 \sim 18)$ & $29(7 \sim 75)$ & $0.006^{*}$ \\
\hline Height $(\mathrm{cm})$ & $85(61 \sim 100)$ & $61(51 \sim 65)$ & $64(55 \sim 90)$ & 0.113 & $63(52 \sim 78)$ & $85(64 \sim 113)$ & $0.009 *$ \\
\hline Weight (kg) & $11.8(5.9 \sim 14)$ & $5.4(3.8 \sim 6.5)$ & $6.1(4.3 \sim 12.5)$ & 0.212 & $5.3(3.5 \sim 8.6)$ & $10.9(6.5 \sim 17.5)$ & $0.019 *$ \\
\hline $\operatorname{BSA}\left(\mathrm{m}^{2}\right)$ & $0.496(0.292 \sim 0.649)$ & $0.277(0.207 \sim 0.327)$ & $0.313(0.238 \sim 0.563)$ & 0.099 & $0.298(0.209 \sim 0.420)$ & $0.505(0.321 \sim 0.742)$ & $0.013 *$ \\
\hline LVEF (\%) & $72.7(63.6 \sim 78.3)$ & $68.8 \%(59.4 \sim 77.0)$ & $71.3(57.1 \sim 87.4)$ & 0.296 & $69.9(57.1 \sim 87.4)$ & $71.3(65.2 \sim 80.8)$ & 0.183 \\
\hline
\end{tabular}

Note: ${ }^{*} P<0.05$

BSA, body surface area; LVEF, left ventricular ejection fraction.

Table 4: Comparison of biomarkers in different groups

\begin{tabular}{|c|c|c|c|c|c|c|c|}
\hline & \multicolumn{3}{|c|}{ Diagnosis } & \multirow[t]{2}{*}{$P$} & \multicolumn{2}{|c|}{ Reversibility } & \multirow[t]{2}{*}{$P$} \\
\hline & Group $1(\mathrm{n}=8)$ & Group $2(n=12)$ & Group $3(n=50)$ & & $\mathrm{RPH}(\mathrm{n}=45)$ & IRPH $(n=14)$ & \\
\hline CECs (\%o) & $\begin{array}{l}0.023 \\
(0.007 \sim 0.065)\end{array}$ & $\begin{array}{l}0.060 \\
(0.007 \sim 0.095)\end{array}$ & $0.094(0.013 \sim 0.341)$ & $0.000^{*}$ & $0.066(0.007 \sim 0.288)$ & $0.112(0.088 \sim 0.341)$ & $0.004 *$ \\
\hline $\begin{array}{l}\text { ET-1 } \\
(\mathrm{pg} / \mathrm{mL})\end{array}$ & $\begin{array}{l}16.535 \\
(7.040 \sim 55.639)\end{array}$ & $\begin{array}{l}61.170 \\
(23.193 \sim 84.745)\end{array}$ & $\begin{array}{l}84.279 \\
(36.730 \sim 145.663)\end{array}$ & $0.000^{*}$ & $\begin{array}{l}76.180 \\
(23.193 \sim 138.680)\end{array}$ & $\begin{array}{l}91.377 \\
(44.324 \sim 145.663)\end{array}$ & 0.065 \\
\hline $\begin{array}{l}\text { eNOS } \\
(\mathrm{ng} / \mathrm{mL})\end{array}$ & $\begin{array}{l}1.302 \\
(0.120 \sim 4.959)\end{array}$ & $\begin{array}{l}6.455 \\
(2.795 \sim 10.312)\end{array}$ & $\begin{array}{l}7.450 \\
(2.567 \sim 12.147)\end{array}$ & $0.000^{*}$ & $\begin{array}{l}7.315 \\
(3.357 \sim 12.148)\end{array}$ & $\begin{array}{l}8.281 \\
(2.567 \sim 10.109)\end{array}$ & 0.301 \\
\hline
\end{tabular}

A



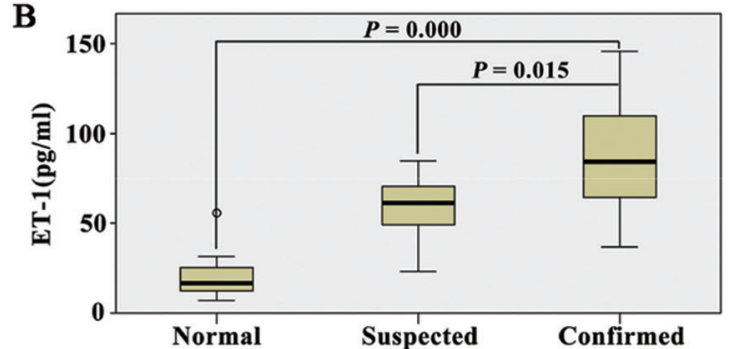

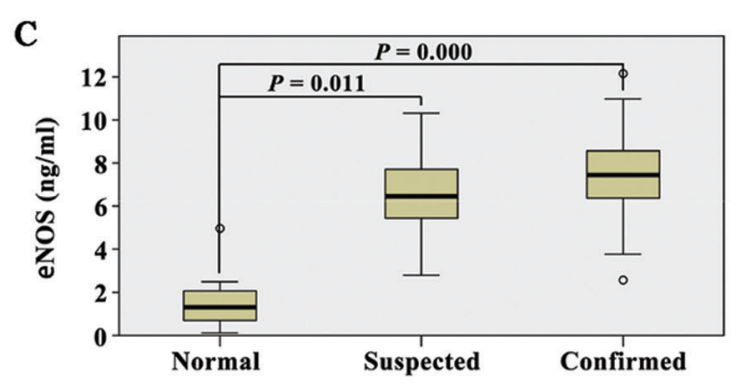

Figure 2: Biomarkers of endothelial injury in the normal, PH-CHD suspected and confirmed groups. A. CECs count was significantly increased in the confirmed group. B. ET-1 level in the confirmed group was higher than that in normal and PH-CHD suspected groups. C. eNOS was higher in the PH-CHD suspected and confirmed groups compared to the normal group 


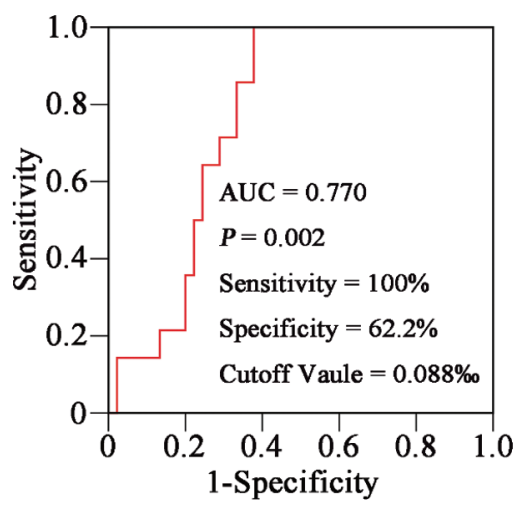

Figure 3: ROC curve of CECs in the diagnosis of IRPH

\subsection{Risk Stratification}

The reversibility of PH-CHD was evaluated using the risk stratification score, and the statistical results showed that the score of the confirmed group was significantly higher than that of the suspected $(P=0.001)$ and normal $(P=0.000)$ groups. Furthermore, the score of the IRPH group was much higher than that of the RPH group. Table 5 shows the score distribution of clinical features combined with CECs and clinical features alone in these two groups. ROC analysis showed that risk stratification was of high value in diagnosing the reversibility of PH-CHD. In addition, compared to the clinical features or CEC count alone for IRPH diagnosis, ROC analysis reached the maximum AUC when clinical features were combined with CECs, and the sensitivity and specificity were relatively high (Fig. 4, Table 6).

Table 5: Score distribution of risk stratification in the two groups

\begin{tabular}{llll}
\hline Risk score & RPH $(\mathrm{n}=45)$ & IRPH $(\mathrm{n}=14)$ & $P$ \\
\hline Clinical features + CECs & $6(4 \sim 10)$ & $9.5(7 \sim 11)$ & $<0.001^{*}$ \\
Clinical features & $5(3 \sim 8)$ & $7.5(5 \sim 9)$ & $<0.001^{*}$ \\
\hline
\end{tabular}
Note: ${ }^{*}<<0.05$.

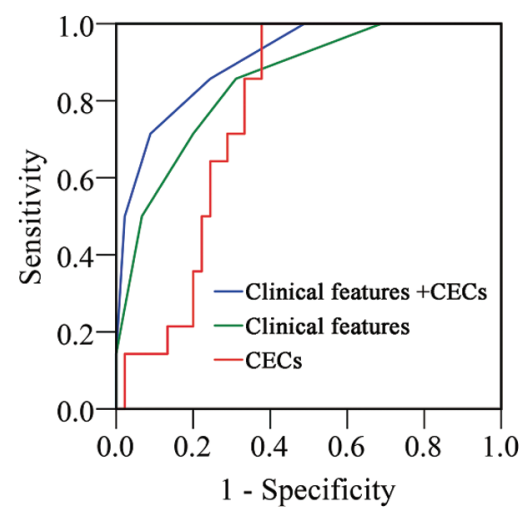

Figure 4: ROC curve for the diagnosis of IRPH 
Table 6: ROC analysis in diagnosing IRPH

\begin{tabular}{llllll}
\hline & AUC & $P$ & Sensitivity & Specificity & Cut-off value \\
\hline Clinical features + CECs & 0.908 & $<0.001$ & $71.4 \%$ & $91.1 \%$ & 7.5 \\
Clinical features & 0.852 & $<0.001$ & $85.7 \%$ & $68.9 \%$ & 5.5 \\
CECs & 0.770 & 0.002 & $100 \%$ & $62.2 \%$ & $0.088 \% 0$ \\
\hline
\end{tabular}

\section{Discussion}

Although the diagnosis, treatment, and prognosis of patients with PH-CHD have greatly improved in recent years, some patients continue to have $\mathrm{PH}$ after surgery, which leads to delayed recovery in the early postoperative period and has a serious impact on the long-term quality of life and lifespan [24-26]. In the clinic, right-heart catheterization is a tool that can be used to evaluate the severity of PH-CHD, but its wide application is limited by the invasive nature of the procedure. Pulmonary endothelial dysfunction plays an important role in the development of PH-CHD. Under normal physiological conditions, vasoactive substances secreted by the pulmonary vascular endothelium are in a circulating compartment. Thus, it provides an opportunity for the noninvasive exploration of vasoactive substances. In the present study, we detected biomarkers of endothelial injury and combined them with clinical features to establish a risk stratification strategy to explore noninvasive methods to evaluate the reversibility of PH-CHD.

With the development of economic and medical technology, the diagnosis of CHD was earlier, and the operation time was shorter than before, which decreased the incidence of IRPH in patients with simple CHD. In the present study, statistical analysis showed that age, height, weight, and BSA were significantly higher in the IRPH group than in the RPH group. In addition, only three of the 21 patients with simple CHD had IRPH in the present study. The remaining patients with IRPH were diagnosed with complex CHD, especially DORV and FSV. The results were similar to those of previous studies and implied that age and diagnosis should be considered when evaluating the reversibility of PH-CHD [27,28].

Endothelin-1 is a powerful intrinsic vasoconstrictor secreted by pulmonary vascular endothelial cells. Studies have found that the expression of ET-1 is increased in PH models, and is closely related to remodel of the pulmonary vascular endothelium [29-31]. Therefore, the endothelial pathway became the main target for PH drug therapy. In this study, the confirmed group showed a significant increase in ET-1 compared to the normal $(P=0.000)$ and suspected $(P=0.015)$ groups. However, there was no significant difference between the IRPH and RPH groups $(P=0.065)$. These results suggested that the expression of ET-1 was up regulated in PH-CHD patients, but it could not be used to evaluate PH reversibility. Other studies also showed that ET-1 was less valuable in prognosis prediction [7,11].

The dysfunction of pulmonary vascular endothelial cells is mainly manifested as the imbalanced expression of ET-1 and NO $[13,14]$. eNOS is a key enzyme in the synthesis of NO. Studies have found a strong time relevance between the concentration of eNOS and the development of $\mathrm{PH}[9,32]$. The present study showed that eNOS was significantly increased in the suspected $(P=0.011)$ and confirmed $(P=$ $0.000)$ groups compared with the normal group. However, there was no difference in eNOS between the suspected and confirmed groups $(P=0.627)$ or between the IRPH and RPH groups $(P=0.301)$. During the period of hyperkinetic $\mathrm{PH}$, endothelium-dependent vasodilation was still preserved, and the secretion of eNOS was increased to relieve PAP and PVR through a compensatory mechanism. At a later stage, EC dysfunction and inhibition of ET-1 reduced the expression of eNOS. Based on the relationship between ET-1 and eNOS, we deduce that the reason why eNOS levels increased in the suspected group was due to the early compensatory mechanism. The patients in the confirmed group might be in the middle-to-late 
stages of $\mathrm{PH}$, which had decreased expression of eNOS. Thus, the results showed that eNOS was of low value in evaluating the reversibility of PH-CHD.

Endothelial cells come into direct contact with blood flow. Therefore, abnormal blood flow is known to impair the structure and function of ECs and activate their apoptosis. Moreover, CECs as a direct reflection of vascular injury are shed from damaged vessels. Smadja and other researchers [7-10] found that CECs were related to the severity of PH-CHD and could be a potential factor in assessing the reversibility of PH-CHD. However, none of them combined this biomarker and patient specific clinical features to explore the feasibility of evaluating IRPH. In the current study, clinical features and biomarkers were combined for risk stratification to assess the reversibility of $\mathrm{PH}-\mathrm{CHD}$, which provided a noninvasive method for clinical decision-making. Statistical analysis showed that CECs in the IRPH group were significantly higher than that in the RPH group. ROC analysis displayed a high sensitivity for CECs in diagnosing the reversibility of PH-CHD, especially when combined with clinical features (Table 6). The risk stratification score was significantly increased in the confirmed and IRPH groups. The ROC curve showed that the risk stratification combining clinical features and CECs was of higher value in evaluating the reversibility of PH-CHD than either factor alone. The results indicated that the combination of clinical features and CECs for risk stratification may be a potentially valuable tool to noninvasively evaluate the reversibility of PH-CHD.

The present study has some limitations. First, considering the invasive nature of the procedure, rightheart catheterization was performed only in highly suspicious IRPH patients in clinical practice. In the present study, only a few patients underwent cardiac catheterization; thus, echocardiographic diagnostic criteria for PH were adopted for grouping. Second, the CECs per milliliter count was very low. Therefore, to ensure the reliability of the experimental data, the transfer of blood samples was minimized during the experiment. However, loss of some CECs was inevitable; hence, the CEC count may be lower than its actual number. Third, blood samples were not collected during the follow-up period, and hence the relationship between the biomarkers and the clinical course is not clear. We hope to address these limitations in future research.

\section{Conclusions}

The CEC count was elevated in patients with PH-CHD and IRPH, and this count showed a high sensitivity in the assessment of PH-CHD reversibility. Although the secretions of ET-1 and eNOS were decreased in PH-CHD patients, their value in the evaluation of PH-CHD reversibility was low. The risk score was significantly increased in the IRPH group, and the risk stratification that combines clinical features with CECs was of high value in identifying IRPH.

Funding Statement: We thank the support of National Nature Science Foundation of China (81771934) and Shanghai Jiao Tong University Medical and Engineering Intersection Fund (YG2019ZDA03).

Conflicts of Interest: There is no conflict of interest for any author.

\section{References}

1. Diller, G. P., Kempny, A., Alonso-Gonzalez, R., Swan, L., Uebing, A. et al. (2015). Survival prospects and circumstances of death in contemporary adult congenital heart disease patients under follow-up at a large tertiary centre. Circulation, 132(22), 2118-2125. DOI 10.1161/CIRCULATIONAHA.115.017202.

2. Martin, G., Jonas, R. (2018). Surgery for congenital heart disease: Improvements in outcomes. American Journal of Perinatology, 35(6), 557-560. DOI 10.1055/s-0038-1639358.

3. van der Feen, D. E., Bartelds, B., de Boer, R. A., Berger, R. (2017). Pulmonary arterial hypertension in congenital heart disease: Translational opportunities to study the reversibility of pulmonary vascular disease. European Heart Journal, 38(26), 2034-2041. DOI 10.1093/eurheartj/ehx034. 
4. Heath, D., Edwards, J. E. (1958). The pathology of hypertensive pulmonary vascular disease: A description of six grades of structural changes in the pulmonary arteries with special reference to congenital cardiac septal defects. Circulation, 18(4), 533-547. DOI 10.1161/01.CIR.18.4.533.

5. Humbert, M., Guignabert, C., Bonnet, S., Dorfmüller, P., Klinger, J. R., Nicolls, M. R. et al. (2018). Pathology and pathobiology of pulmonary hypertension: State of the art and research perspectives. European Respiratory Journal, 53(1), 1801887. DOI 10.1183/13993003.01887-2018.

6. Sallmon, H., Hatch, A., Murthy, S. K., Plouffe, B. D., Hansmann, G. (2017). Circulating endothelial cell quantification by microfluidics chip in pulmonary arterial hypertension. American Journal of Respiratory Cell and Molecular Biology, 56(5), 680-682. DOI 10.1165/rcmb.2017-0026LE.

7. Latus, H., Karanatsios, G., Basan, U., Salser, K., Müller, S., Khalil, M. et al. (2016). Clinical and prognostic value of endothelin-1 and big endothelin-1 expression in children with pulmonary hypertension. Heart, 102(13), 1052 1058. DOI 10.1136/heartjnl-2015-308743.

8. Li, X., Qiu, J., Pan, M., Zheng, D., Zhu, J. (2015). Correlation between congenital heart disease complicated with pulmonary artery hypertension and circulating endothelial cells as well as endothelin-1. International Journal of Clinical and Experimental Pathology, 8(9), 10743-10751.

9. Szulcek, R., Happé, C. M., Rol, N., Fontijn, R. D., Dickhoff, C. et al. (2016). Delayed microvascular shear adaptation in pulmonary arterial hypertension role of platelet endothelial cell adhesion molecule-1 cleavage. American Journal of Respiratory and Critical Care Medicine, 193(12), 1410-1420. DOI 10.1164/rccm.201506-1231OC.

10. Smadja, D. M., Gaussem, P., Mauge, L., Israel-Biet, D., Dignat-George, F. et al. (2009). Circulating endothelial cells: A new candidate biomarker of irreversible pulmonary hypertension secondary to congenital heart disease. Circulation, 119(3), 374-381. DOI 10.1161/CIRCULATIONAHA.108.808246.

11. Montani, D., Souza, R., Binkert, C., Fischli, W., Simonneau, G. et al. (2007). Endothelin-1/endothelin-3 ratio: A potential prognostic factor of pulmonary arterial hypertension. Chest, 131(1), 101-108. DOI 10.1378/chest.06-0682.

12. Rubens, C., Ewert, R., Halank, M., Wensel, R., Orzechowski, H. D. et al. (2001). Big endothelin-1 and endothelin-1 plasma levels are correlated with the severity of primary pulmonary hypertension. Chest, 120(5), 1562-1562. DOI 10.1378/chest.120.5.1562.

13. Dai, Z. K., Tan, M. S., Chai, C. Y., Yeh, J. L., Chou, S. H. et al. (2004). Upregulation of endothelial nitric oxide synthase and endothelin-1 in pulmonary hypertension secondary to heart failure in aorta-banded rats. Pediatric Pulmonology, 37(3), 249-256. DOI 10.1002/ppul.10413.

14. Hoehn, T., Stiller, B., Mcphaden, A. R., Wadsworth, R. M. (2009). Nitric oxide synthases in infants and children with pulmonary hypertension and congenital heart disease. Respiratory Research, 10(1), 110. DOI 10.1186/14659921-10-110.

15. Farinacci, M., Krahn, T., Dinh, W., Volk, H. D., Düngen, H. D. et al. (2019). Circulating endothelial cells as biomarker for cardiovascular diseases. Research and Practice in Thrombosis and Haemostasis, 3(1), 49-58. DOI 10.1002/rth2.12158.

16. Feen, D., Bartelds, B., Boer, R., Berger, R. (2018). Assessment of reversibility in pulmonary arterial hypertension and congenital heart disease. Heart, 105(4), 276-282. DOI 10.1136/heartjnl-2018-314025.

17. Kulik, T. J. (2012). Pulmonary blood flow and pulmonary hypertension: Is the pulmonary circulation flowophobic or flowophilic? Pulmonary Circulation, 2(3), 327-339. DOI 10.4103/2045-8932.101644.

18. O'Blenes, S. B., Fischer, S., McIntyre, B., Keshavjee, S., Rabinovitch, M. (2001). Hemodynamic unloading leads to regression of pulmonary vascular disease in rats. Journal of Thoracic and Cardiovascular Surgery, 121(2), 279-289. DOI $10.1067 / \mathrm{mtc} .2001 .111657$.

19. Simonneau, G., Montani, D., Celermajer, D. S., Denton, C. P., Gatzoulis, M. A. et al. (2018). Haemodynamic definitions and updated clinical classification of pulmonary hypertension. European Respiratory Journal, 53(1), 1801913. DOI 10.1183/13993003.01913-2018.

20. Galiè, N., Humbert, M., Vachiery, J. L., Gibbs, S., Lang, I. et al. (2016). 2015 ESC/ERS guidelines for the diagnosis and treatment of pulmonary hypertension: The joint task force for the diagnosis and treatment of pulmonary hypertension of the european society of cardiology (ESC) and the european respiratory society (ERS): Endorsed by: Association for european paediatric and congenital cardiology (AEPC), international 
society for heart and lung transplantation (ISHLT). European Heart Journal, 37(1), 67-119. DOI 10.1093/ eurheartj/ehv317.

21. Lanuti, P., Simeone, P., Rotta, G., Almici, C., Avvisati, G. et al. (2018). A standardized flow cytometry network study for the assessment of circulating endothelial cell physiological ranges. Scientific Reports, 8(1), 5823. DOI 10.1038/s41598-018-24234-0.

22. Řádek, M., Babuňková, E., Špaček, M., Kvasnička, T., Kvasnička, J. (2019). Determination of circulating endothelial cells and endothelial progenitor cells using multicolor flow cytometry in patients with thrombophilia. Acta Haematologica, 142(2), 113-119. DOI 10.1159/000499524.

23. Biasi, S. D., Gibellini, L., Feletti, A., Pavesi, G., Bianchini, E. et al. (2017). High speed flow cytometry allows the detection of circulating endothelial cells in hemangioblastoma patients. Methods, 134-135, 3-10. DOI 10.1016/j. ymeth.2017.11.002.

24. Alessandra, M., Massimiliano, P., Enri, L., Bacchi, R. M. L., Angelo, B. et al. (2014). Current era survival of patients with pulmonary arterial hypertension associated with congenital heart disease: A comparison between clinical subgroups. European Heart Journal, 35(11), 716-724. DOI 10.1093/eurheartj/eht072.

25. Barst, R. J., Mcgoon, M. D., Elliott, C. G., Foreman, A. J., Miller, D. P. et al. (2012). Survival in childhood pulmonary arterial hypertension: Insights from the registry to evaluate early and long-term pulmonary arterial hypertension disease management. Circulation, 125(1), 113-122. DOI 10.1161/CIRCULATIONAHA.111.026591.

26. Hansmann, G. (2017). Pulmonary hypertension in infants, children, and young adults. Journal of the American College of Cardiology, 69(20), 2551. DOI 10.1016/j.jacc.2017.03.575.

27. Hjortshoj, C. S., Jensen, A. S., Sorensen, K., Nagy, E., Johansson, B. et al. (2017). Epidemiological changes in eisenmenger syndrome in the nordic region in 1977-2012. Heart, 103(17), 1353-1358. DOI 10.1136/heartjnl2016-310979.

28. Ntiloudi, D., Zanos, S., Gatzoulis, M. A., Karvounis, H., Giannakoulas, G. (2019). How to evaluate patients with congenital heart disease-related pulmonary arterial hypertension. Expert Review of Cardiovascular Therapy, 17(1), 11-18. DOI 10.1080/14779072.2019.1550716.

29. Thenappan, T., Ormiston, M. L., Ryan, J. J., Archer, S. L. (2018). Pulmonary arterial hypertension: Pathogenesis and clinical management. BMJ, 360, j5492. DOI 10.1136/bmj.j5492.

30. Agnoletti, G., Gala, S., Ferroni, F., Bordese, R., Appendini, L. et al. (2017). Endothelin inhibitors lower pulmonary vascular resistance and improve functional capacity in patients with fontan circulation. Journal of Thoracic and Cardiovascular Surgery, 153(6), 1468-1475. DOI 10.1016/j.jtcvs.2017.01.051.

31. Mehta, S., Sastry, B., Souza, R., Torbicki, A., Ghofrani, H. A. et al. (2017). Macitentan improves health-related quality of life for patients with pulmonary arterial hypertension: Results from the randomized controlled seraphin trial. Chest, 151(1), 106-118. DOI 10.1016/j.chest.2016.08.1473.

32. Bharath, L. P., Cho, J. M., Park, S. K., Ruan, T., Symons, J. D. (2017). Endothelial cell autophagy maintains shear stressinduced nitric oxide generation via glycolysis-dependent purinergic signaling to endothelial nitric oxide synthase. Arteriosclerosis Thrombosis and Vascular Biology, 37(9), 1646-1656. DOI 10.1161/ATVBAHA.117.309510. 